\title{
Agôn
}

Revue des arts de la scène

Critiques | Saison 2013-2014

\section{Invisibles. La tragédie des Chibanis}

Une création de Nasser Djemaï

\section{Maëline Le Lay}

\section{Q OpenEdition}

Journals

Édition électronique

URL : http://journals.openedition.org/agon/2672

DOI : 10.4000/agon.2672

ISSN : 1961-8581

Éditeur

Association Agôn

Référence électronique

Maëline Le Lay, «Invisibles. La tragédie des Chibanis », Agôn [En ligne], Critiques, mis en ligne le 04 juillet 2013, consulté le 23 septembre 2020. URL : http://journals.openedition.org/agon/2672 ; DOI https://doi.org/10.4000/agon.2672

Ce document a été généré automatiquement le 23 septembre 2020

Association Agôn et les auteurs des articles 


\title{
Invisibles. La tragédie des Chibanis
}

\author{
Une création de Nasser Djemaï
}

Maëline Le Lay

\section{RÉFÉRENCE}

Invisibles. La tragédie des Chibanis de Nasser Djemaï. Créé le 22 novembre 2011 à la MC2, Grenoble

Tournée en France en 2012-2013

Informations complémentaires : http://nasserdjemai.com/invisibles_01.htm

1 Invisibles, la dernière pièce du comédien, auteur et metteur en scène Nasser Djemaï, remporte depuis le début de sa tournée un franc succès auprès du public. Comme ses deux précédentes créations -Les vipères se parfument au jasmin - et L'étoile de NoëlInvisibles est un texte à coloration autobiographique. Il a pour point de départ la vie du père de l'auteur, un chibani (qui signifie «cheveux blancs» en arabe), un de ces immigrés algériens venus en France dans la période de l'après-guerre pour y travailler sur des chantiers de construction, à Marseille d'abord, puis à Grenoble. Djemaï poursuit donc avec Invisibles une thématique qui traverse les trois pièces composant son œuvre : l'exil, l'immigration, l'expérience de l'Autre à travers la rencontre mais aussi (et surtout) au prisme de la xénophobie et de l'oppression.

2 Invisibles raconte l'histoire de Martin, un jeune homme qui se met en quête de son père inconnu, muni des maigres indices chuchotés par sa mère avant de rendre son dernier souffle : une adresse, deux noms, un coffret à remettre à un certain El Hadj. Il se retrouve dans un foyer de migrants d'Afrique du Nord retraités où il est accueilli par quatre Chibanis qui lui apprennent que le fameux El Hadj est frappé d'aphasie. Au fil des discussions avec les Chibanis qui s'occupent de El Hadj, il découvrira l'âpreté de leur quotidien et remontera avec eux les traces de son passé jusqu'aux origines de sa naissance. 
Invisibles, photographie de scène

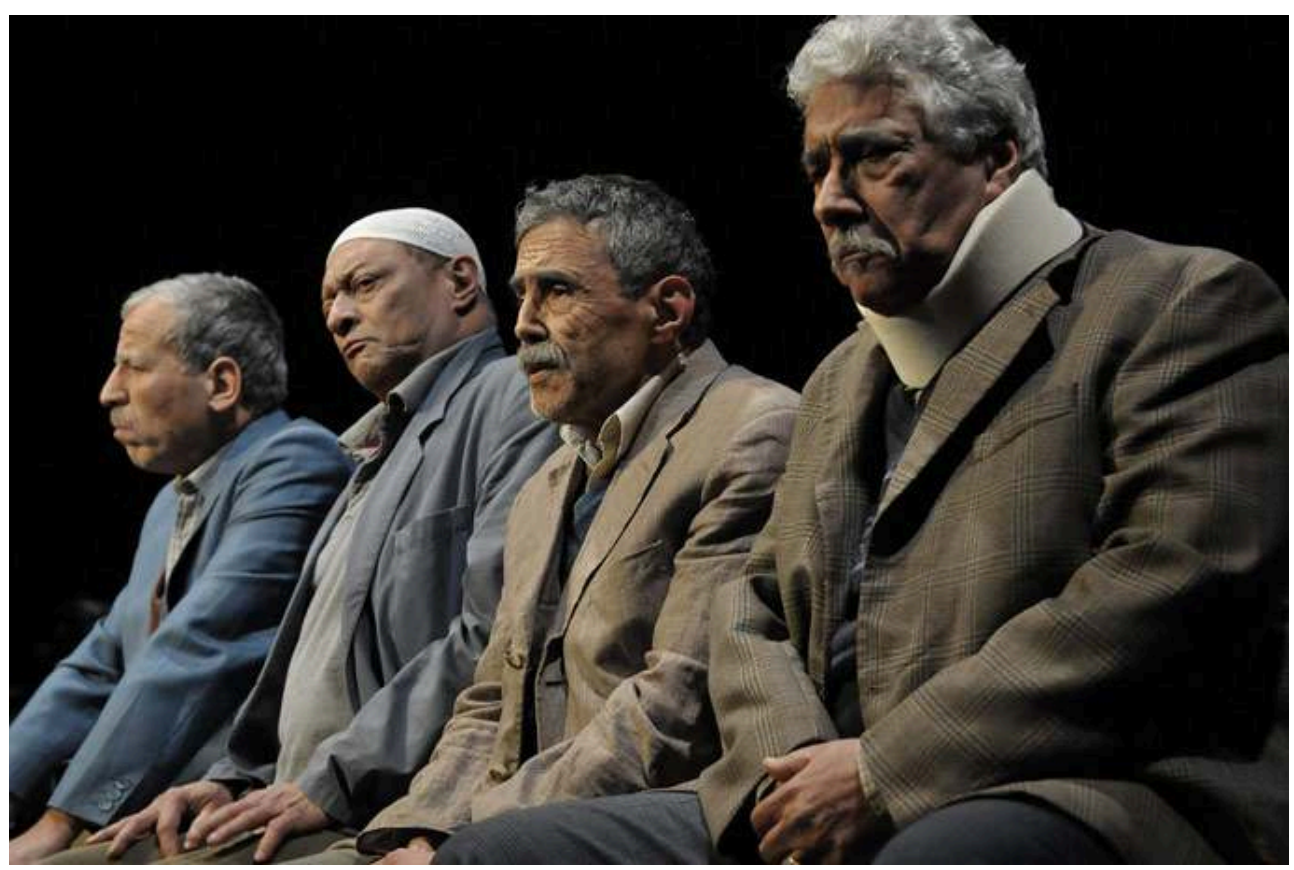

(C)Philippe Delacroix

3 Il faut d'abord saluer l'important investissement de l'auteur dans ce travail sur les Chibanis. Loin de se contenter de ses souvenirs d'enfance, Nasser Djemaï est allé à leur rencontre dans des foyers de migrants retraités et s'est longuement entretenu avec eux. De ce travail qui relève clairement de l'ethnographie, mais aussi de récits d'enfance et de la lecture d'essais critiques et de documentaires, est née la création dramatique. Ce processus d'écriture témoigne de la prudence de l'auteur face à cette thématique à la fois proche et lointaine. Il y a un réel souci chez Nasser Djemaï de ne pas usurper la parole de ces hommes et, en quelque sorte, de trouver la juste distance entre lui-même et son sujet de création. Ce recul critique qu'il déploie dans de nombreux discours ou écrits est tout à fait louable ; il est la marque d'un écrivain à l'œil lucide et au geste généreux, d'un artiste qui n'hésite pas à se frotter aux aspérités du monde et aux blessures de l'Histoire.

4 Par ailleurs, il faut rendre gré à l'auteur de traiter d'un tel thème dans l'ambiance politique actuelle où la mode est à la traque des étrangers et à la criminalisation des " assistés ». Dans ce climat où nous baignons, tendu par la xénophobie, il est salutaire d'entendre la voix de ces «invisibles » qui sont aussi parfois muets et résignés et à qui Nasser Djemaï prête par instants fulgurants le grondement de la révolte et de l'indignation. D'aucuns pourraient considérer que la volonté de trop en dire dessert le propos mais d'autres estimeront qu'elle est partie intégrante du discours indigné, comme l'est celui porté par le rap militant par exemple.

5 Même si l'auteur affirme avoir voulu éviter à tout prix de verser dans le témoignage documentaire pour donner toute sa place à la poésie et au théâtre, son texte n'en demeure pas moins testimonial et pourrait même, au regard de l'émulation intellectuelle et politique qu'il a suscité, se lire comme une sorte de plaidoyer pour les Chibanis. Comment d'ailleurs aurait-il pu en être autrement après un tel travail 
d'enquête? Mais ce refus du réalisme ne devrait pas pour autant l'empêcher de rendre l'histoire un tant soit peu crédible. Ce n'est hélas pas tout à fait le cas.

6 Si cette pièce n'est pas donc certainement pas dénuée d'intérêt dans son projet et son intention, elle souffre néanmoins d'un certain nombre de maladresses formelles et d'un manque de cohérence qui amoindrissent son propos. Le spectateur est dès le début mis à l'écart du héros et de sa quête car il comprend immédiatement que le père de Martin n'est autre que $\mathrm{El} \mathrm{Hadj.} \mathrm{La} \mathrm{compréhension} \mathrm{réciproque} \mathrm{par} \mathrm{le} \mathrm{père} \mathrm{et} \mathrm{le} \mathrm{fils} \mathrm{de} \mathrm{leur} \mathrm{lien}$ de parenté qui ne survient qu'à la fin, est donc artificielle et tombe à plat après deux heures de spectacle et plusieurs dizaines de pages d'indices tout à fait explicites. De même, les crises que subit fréquemment Martin dès qu'il tente de sortir du foyer font tout autant factices et participent au mélo dont la pièce est par endroits affectée. La voix de la défunte Louise qui scande le texte comme une litanie plaintive, sommant son fils de partir à la découverte de son « destin » et surtout la scène finale d'embrassades collectives où Martin après avoir longuement enlacé son père s'assoit sur les genoux de chacun des Chibanis, font basculer l'histoire dans un style pathos ennuyeux.

7 Mais c'est dans la gestion linguistique des langages affectés aux personnages que l'artifice et le manque de réalisme sont les plus manifestes. On sait quel exercice délicat représente la transcription d'un sociolecte - notamment lorsque celui-ci est en partie caractérisé par la maîtrise incertaine du français - mais s'il est un registre dans lequel un dramaturge est censé exceller, c'est bien celui du langage verbal et c'est hélas sur ce point que la création de Nasser Djemaï me semble la moins réussie.

8 Il précise dans ses indications de mise en scène que la pièce doit être jouée dans « un mélange de français et d'arabe » mais elle n'est écrite qu'en français et dans le jeu dirigé par l'auteur, ne laisse que peu de place à l'arabe, tout juste pour les interjections. Par ailleurs, l'usage assumé de certaines tournures syntaxiques non-orthodoxes ${ }^{1}$ de renvoi, comme un écho gênant, au français "petit-nègre " ou "tirailleur » tel que véhiculé dans la littérature coloniale. On perçoit bien la tentative de donner à entendre un tel sociolecte mais le résultat est maladroit et l'on se demande où est passée la poésie que cherchait à instiller l'auteur dans ce texte ${ }^{2}$.

9 La pesanteur et l'artifice de l'expression des Chibanis ainsi que la tonalité pathétique de plusieurs scènes ne parviennent cependant pas à gâcher les deux moments les plus justes de la pièce condensés dans les tableaux 9 et 19, comme deux souffles d'émotion délicate qui rythment la pièce en son milieu et sa fin. Le tableau 9 est composé du bref monologue de Majid sur l'invisibilité où il raconte avec tact et retenue le souvenir du contact fugace sur son épaule d'une femme inconnue éplorée et blottie contre lui sur le banc d'un parc. Dans le tableau 19 s'entrecroisent les voix de Martin et de Driss, chacun perdu dans le souvenir douloureux d'un amour filial et paternel impossible.

10 Sur la scène donc, les voix se croisent sans se répondre, à l'image peut-être de la relation qu'entretiennent ces hommes avec leur pays d'adoption, l'impression d'être ignorés, de ne pas exister aux yeux de la société française ${ }^{3}$. Nasser Djemaï entendait donner de la visibilité à ces hommes délaissés pris dans ce non-choix, cet exil à vie comme l'exprime simplement Hamid : «Tu sais bien pourquoi on reste tous ici! Si je rentre au pays, je perds mon complément retraite, et comment je fais pour vivre avec rien, moi ?».

11 L'invisibilité renvoie donc de prime abord à l'anonymat mais dans sa préface, l'auteur se réfère au mythe grec d'Hadès (qui signifie "invisible») dans lequel celui qui parviendrait aux enfers, royaume d'Hadès, pourrait interroger ses ancêtres et revenir 
dans le monde des vivants à condition de ne pas s'asseoir sur la chaise d'oubli. La mise en scène finale obéit à cette injonction mythologique puisque Martin à qui elle est adressée, s'assoit sur les genoux de chacun de ces hommes qui se souviennent du passé de El Hadj.

12 Outre les clichés et le pathos dans lequel la pièce a tendance à sombrer, l'absence de tension dramatique est indubitablement ce qui lui fait le plus défaut. Le faux suspense de la révélation familiale auquel s'ajoute l'ensemble de scènes diverses du foyer inspirées des notes d'observation sociologique de l'auteur mais que l'on sent comme « collées » et non liées entre elles, les scènes 5 - présumées oniriques - tissées de voix d'outre-tombe et de voix téléphoniques, viennent alourdir l'ensemble textuel et scénique au lieu de le poétiser.

\section{NOTES}

1. Relevons notamment la postposition des pronoms COI («les métiers à nous») et la substitution des pronoms relatifs par les pronoms personnels («La porte elle est fermée. Je protège toi»).

2. Ce n'est sans doute pas non plus ainsi qu'avait procédé Kateb Yacine avec le français et l'arabe dans Mohamed, prends ta valise notamment, mais l'option linguistique retenue pour cette pièce comme pour les autres du théâtre politique de Kateb Yacine présentait le mérite d'être plus claire: une écriture manifestement bilingue et dépouillée dans chacune des langues. Le rendu en français (à partir de traductions faites par l'auteur lui-même, les comédiens, sa femme et son fils) est aussi plus limpide et se situe aux antipodes de la " pose » traduit par ce curieux langage que prête Nasser Djemaï à ses personnages.

3. Si le dilemme et la tragédie des Chibanis sont justement exprimés à différents endroits du texte, il faut toutefois rappeler que le phénomène social auquel ils renvoient n'a rien de nouveau dans le monde de la littérature et des arts de la scène. D'autres auteurs en avaient traité avant lui et sans doute avec plus de justesse et d'authenticité. Citons à titre d'exemple Yamina Benguigui qui avait pris le parti d'en faire un vrai reportage dans Mémoires d'immigrés et Tahar Ben Jelloun dans les deux essais tirés de sa thèse de doctorat sur les émigrés nord-africains: La Réclusion solitaire, texte de prose poétique en 1976 et La plus haute des solitudes, un essai documentaire paru en 1977. 